J. clin. Path. (1949), 2, 250.

\title{
THE PLATE VIRULENCE TEST FOR DIPHTHERIA
}

\author{
BY \\ STEPHEN D. ELEK \\ From the Department of Bacteriology, St. George's Hospital \\ Medical School, London*
}

(RECEIVED FOR PUblication, MAY 20, 1949)

A method has recently been described (Elek, 1948) for the detection in vitro of toxin-producing organisms including $C$. diphtheriae. The present paper has two purposes. The first is the examination of a larger series of strains of $C$. diphtheriae with a view to establishing the reliability of the procedure, for if the method is to be of any practical value for clinical purposes it must demonstrate all toxin-producing strains within a reasonably short time. The second purpose is the standardization of the ingredients used so as to yield the best results and to ensure that the conditions of the test may be accurately reproducible in different laboratories.

Petrie and Steabben (1943) first suggested the use of nutrient media as a matrix for toxinantitoxin reactions. They evolved a method for the detection of toxicogenic clostridia and briefly reported some experiments with diphtheria bacilli. Ouchterlony (1948) showed that when diphtheria antitoxin was incorporated in a serum ditch plate and a toxin-producing strain streaked across, several lines were produced, one of which was caused by the toxin. For the practical test of his in vitro method he incorporated various concentrations of antitoxin in serum agar plates, as did Petrie and Steabben (1943), and each strain was tested on a series of plates with falling antitoxin concentrations. The appearance of a halo around the inoculum was regarded as positive. Two series of strains were examined by this method and by the subcutaneous guinea-pig test. In the first series of 237 strains there was a discrepancy amounting to $8.5 \%$. Of the guinea-pig positive strains $5.1 \%$ failed to give a halo, and $3.4 \%$ of avirulent strains gave false positives. In the second series of $\mathbf{3 0 8}$ strains the discrepancy was $6.2 \%$, and included $4.2 \%$ false positives. Elek (1948) criticized Petrie

\footnotetext{
*Part of Ph.D. (London) thesis submitted July, 1948.
}

and Steabben's method on the grounds that a ring in a serum plate does not necessarily signify a toxin-antitoxin reaction and described a new tech- $\vec{Z}$ nique using a medium better suited for toxin production. The antitoxin gradient was set up by means of a filter paper strip dipped into highly con- $\frac{3}{\sigma}$ centrated refined antitoxin. This system was found $\stackrel{\mathbb{C}}{-}$ to be free from the zone effect and the theoretical $\overrightarrow{0}$ considerations are dealt with in a later paper (Eles, $\overrightarrow{0}$ in press). In a small series complete agreement was found between the in vivo and in vit methods, and furthermore the results could read within 24 to 48 hours compared with the 48 to 96 hours required by the method using serial $\frac{\otimes}{\varnothing}$ plates containing graded quantities of antitoxin. Carter and Wilson (1949), using Elek's method but substituting human serum for horse serum, found absolute agreement with 200 strains between the in vivo and in vitro tests. Ouchterlony (1949) in a later paper reports the use of serum ditch plates.옹 Readings were taken at 24-hour intervals for up to $:$ four days.

Concerning the constituents of a medium $\hat{\delta}^{\circ}$ designed to give a good yield of toxin much $₹$ information is already available. Almost all the ? work however was carried out with the classical $\supset$ Park-Williams No. 8 strain, and it is by no means certain that a medium giving optimal toxin produc- $\bar{N}$ tion with it will necessarily meet the requirements $\sigma$ of all other toxin-producing strains. Until about $N$ 25 years ago it was widely believed that only meat $\underset{\omega}{N}$ infusion media could be used for the culture of 0 diphtheria bacilli. Davis and Ferry (1919) stated 0 that it was impossible to obtain growth unless $\underset{\mathcal{D}}{\overparen{D}}$ $0.2 \%$ meat infusion was present, and $10 \%$ was $\stackrel{\odot}{+}$ needed for toxin production. Wadsworth and $\square$ Wheeler (1928) succeeded in producing toxin on $\stackrel{\vec{D}}{\circ}$ peptone without meat infusion, but with various $\stackrel{\varnothing}{\varnothing}$ chemicals added. The addition of glucose to $\stackrel{\mathbb{Q}}{\varrho}$ 
infusion media as a source of energy was suggested by various authors (Park and Williams, 1896; Smith, 1899; Locke and Main, 1928 ; Ramon, 1929 ; Hazen and Heller, 1931) and sodium lactate was introduced by Wadsworth and Wheeler in 1928. Pope (1932a) in a very careful reinvestigation of the effect of various carbohydrates and organic acids found that a concentration of $0.6 \%$ lactic acid was optimal. He used proteose-peptone for his media and the Park-Williams No. 8 strain for toxin produztion. He concluded that acclimatization to the medium was unimportant, but severe heat treatment, such as autoclaving of the medium, would destroy toxin production without interfering with growth. Pope and Healey (1933a and b) examined maltose concentrations and found $0.4 \%$ to be the optimum, $0.8 \%$ yielding decidedly less toxin. They found further that the best growth of the organism did not necessarily yield the maximum amount of toxin, but that this varied with the medium. They also established the very important fact that the initial presence of the toxin was without effect on growth and further toxin production. Finally they proved that toxin production was maximal, other things being equal, when growth occurred at the air/liquid surface. The results of these observations were incorporated in the medium previously described (Elek, 1948).

\section{Experimental Work and Materials}

Subsequent work has shown that successive batches of the medium showed some variation, and slight modifications have been made. Charcoal-clearing has been omitted, as filtering through paper pulp resulted in a sufficiently clear medium. The method of adjusting the reaction had to be standardized as this stage determines the precipitation of phosphates, and consequently the iron content of the medium. The preparation of the medium was accordingly modified as follows.

Twenty grammes of proteose-peptone ("difco"), $3 \mathrm{~g}$. of maltose, and $0.7 \mathrm{ml}$. of lactic acid (B.P.) were dissolved in $500 \mathrm{ml}$. of distilled water. To this $1.5 \mathrm{ml}$. of $40 \% \mathrm{NaOH}$ was added and the medium well shaken and heated to boiling point. The deposit was filtered off through filter paper and the reaction adjusted to $p \mathrm{H} 7.8$ by adding normal $\mathrm{HCl}$. Then $500 \mathrm{ml}$. of $3 \%$ agar (powder) were prepared in $1 \% \mathrm{NaCl}$ and its reaction adjusted to $p \mathrm{H}$ 7.8. This was filtered through paper pulp in a Buchner funnel and then added to the fluid base. After distribution in $10 \mathrm{ml}$. quantities it was sterilized by autoclaving for 10 minutes at $10 \mathrm{lb}$.

To ensure an even thickness of the medium special compressed flint-glass petri dishes, $3 \frac{1}{2}$ in. in diameter, were used. As these are mould-compressed the bottoms are absolutely flat, and this combined with a standard amount of medium for pouring $(12 \mathrm{ml}$.) gives an even thickness in all the plates. The plates were poured on a levelling board supported on three points and set horizontal with a spirit level. For routine purposes neither the mould-compressed plates nor the levelling board is absolutely necessary. Commercial refined diphtheria antitoxin globulins were used diluted with sterile carbol-saline $(0.5 \%$ phenol in normal saline) to a concentration of 1,000 units per $\mathrm{ml}$. This preparation of antitoxin contains $0.35 \%$ of cresol, but as this does not diffuse out it does not

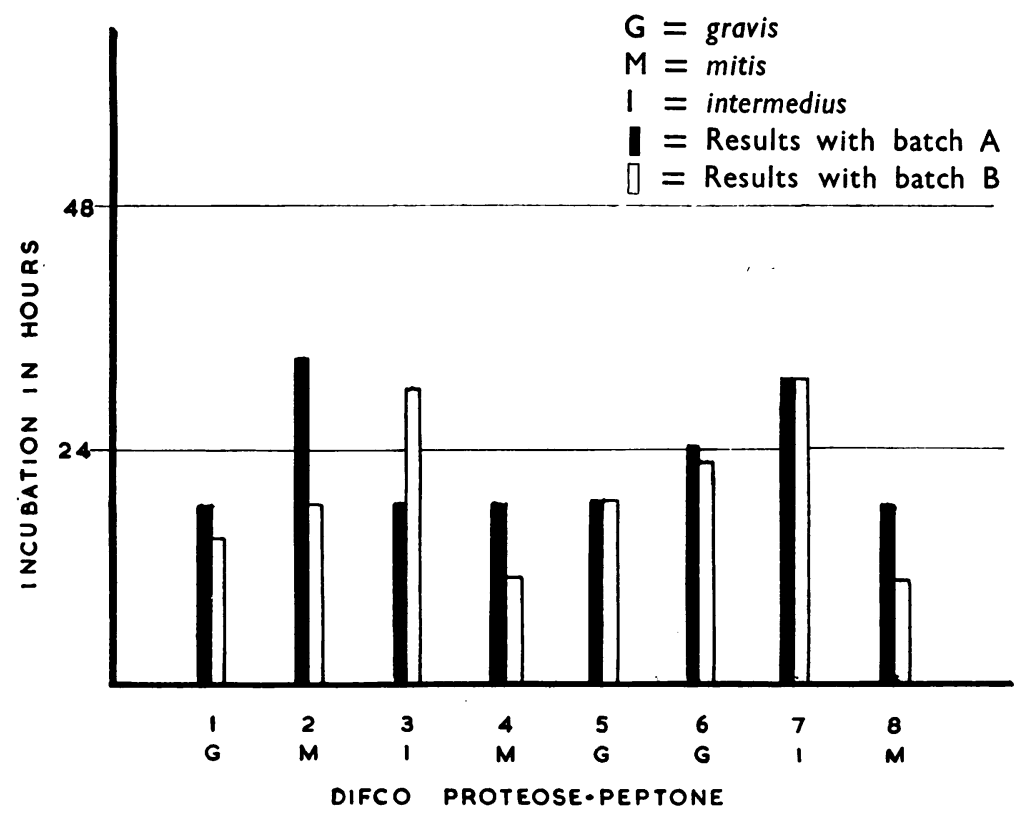

interfere with the reaction. In fact dilution with carbol-saline is useful as it reduces the risk of contamination. A filter strip measuring $60 \mathrm{~mm}$. by $15 \mathrm{~mm}$. sterilized by dry heat was moistened with the antitoxin by immersing it completely or dropping the antitoxin on to it. Plates were poured with $10 \mathrm{ml}$. of the medium and $2 \mathrm{ml}$. of normal horse serum and the filter strip was embedded in the still fluid medium leaving an unbroken surface for inoculation. Two brands of maltose were tried. One was turer's name and the other was "difco." They gave identical re-

Fig. 1.-Comparison, on the basis of the time required for positive results, of two batches of medium (strains 1 to 8). an old batch without a manufac- 
sults. Two samples of lactic acid were tried, and although both complied with B.P. requirements, only one gave satisfactory results, no lines being produced with the other within 48 hours.

Method for Assessing Media.-It was found that different batches thus prepared did not show appreciable variations. Furthermore, the time of appearance of the toxin-antitoxin line for a given organism on different batches was roughly the same. Fig. 1 shows a set of experiments illustrating this point. Two identical batches of media were prepared with proteose-peptone ("difco") and the same batch of horse serum was used for enrichment of both. Eight virulent strains of $C$. diphtheriae were used consisting of three gravis, three mitis, and two intermedius strains. The tests were carried out in the ordinary fashion by streaking each organism at right angles to the filter strip. The width of the streak was not standardized beyond using the same loop. Readings were taken at three-hourly intervals, day and night, for 48 hours and the earliest appearance of the lines recorded. The maximum time difference for any one strain on the two media was about 15 hours, and the average about five hours. Clearly the time of appearance of the lines can serve as a rough measure of the suitability of the medium for toxin production. In this way a comparison can be made, for instance, between the efficacy, for the purposes of this test, of various commercial peptones.

Choice of Peptone. -It is well known that media prepared with different commercial peptones show great variation in toxin production. Thus Hosoya, Ozawa, and Tanaka (1933) found that Chapoteau peptone was highly

FIG. 2.-Effect of various peptones on toxin production. Suitability of the peptone for the purposes of the test is inversely related to the height of the lines. Strains 1 to 8 identical with those in Fig. 1. potent, yielding a high lethal dose and Lf 10.5, whereas the same medium prepared with Witte's peptone or Teruuchi peptone gave toxins of low potency only. With "difco" proteose-peptone Ando and Komiyama (1935) obtained yields of Lf 18 or more, thus confirming Pope's results. Apart from "difco" proteose-peptone (Fig. 1), five further brands of peptone were tested by substituting them in the preparation of the medium described. These were "difco" peptone, "eupeptone No. 1," Evans peptone, "oxoid," and Witte's peptone. All the media were prepared in exactly the same manner and the same eight strains were used for all these experiments. The same batch of serum (serum A in Fig. 3) was used throughout. The plates were incubated for five days, readings being taken three-hourly day and night for the first 60 hours, and thereafter every 12 hours. Fig. 2 shows the results obtained, the height of the lines representing the incubation necessary to obtain a positive result. An, arrow indicates absence of line production within the five days of the experiment. It will be seen that two of the peptones, "difco" and Evans, gave positives with all the eight strains well

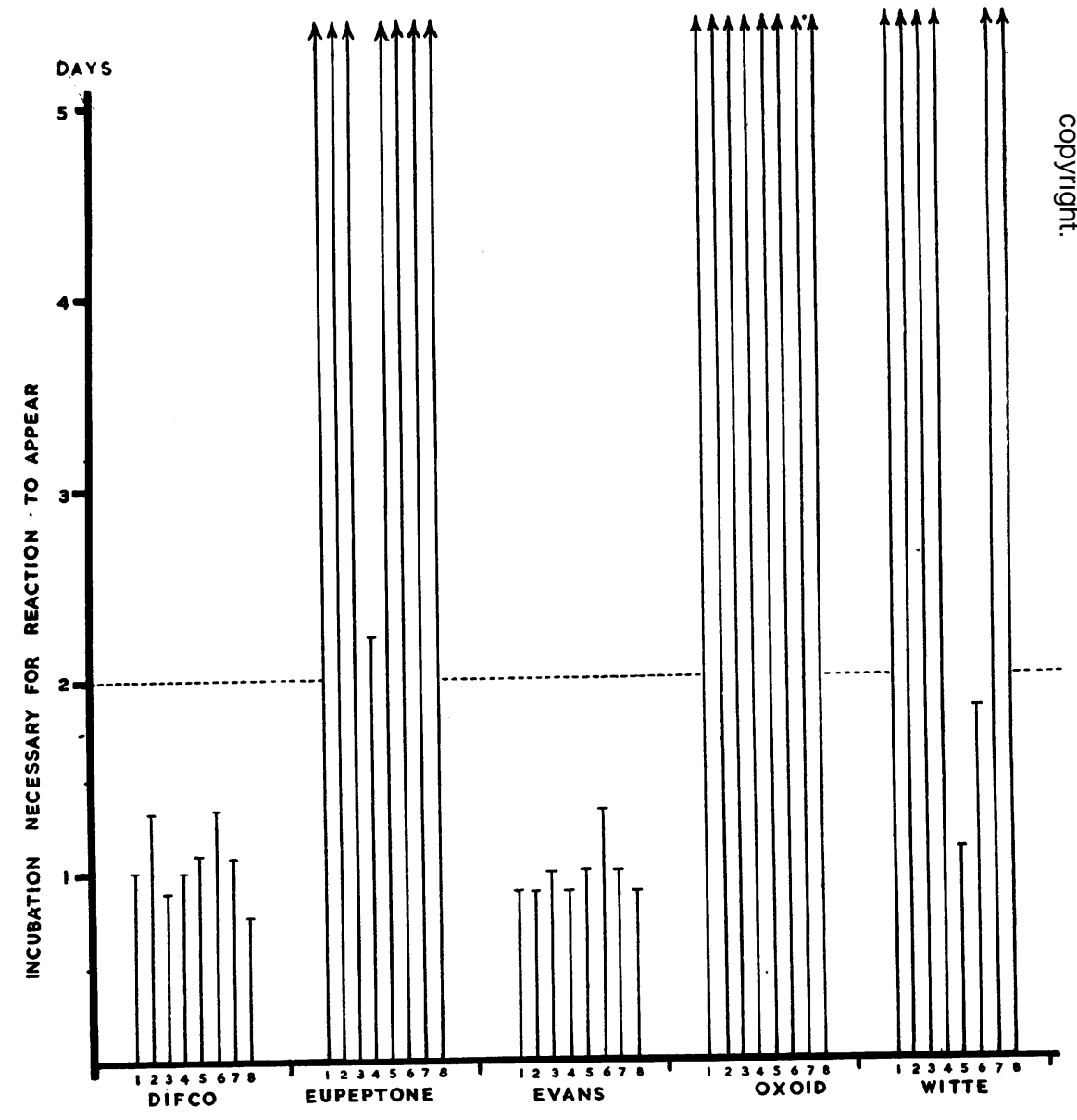


within 48 hours. The general impression one gains is that neither is quite as potent as the "difco" proteose-peptone (compare Fig. 1). Evans peptone, however, is almost as good, and "difco" peptone comes third. "Eupeptone No. 1," "oxoid" and Witte's peptone are clearly unsuitable for the purposes of this test. Witte's peptone gave positives with only two strains within 48 hours (both gravis). The other two peptones gave no positives in two days, the time within which all virulent strains gave positive results with proteose-peptone. Evans peptone is probably suitable for the test, but testing with a much larger series is required. So far this has been done only with "difco" proteose-peptone.

Iron and Copper Content.-Locke and Main (1930) found that media usually used for toxin production contained about 1-4 mg. of copper per litre, and about 0.1 to $0.4 \mathrm{mg}$. of iron per litre. Pope (1932b) reinvestigating the problem found that the addition of copper to media had little effect as long as it did not exceed 8 to $10 \mathrm{mg}$. per litre, which was the maximum tolerated. He also found that filtered medium was improved by iron, but media sterilized by heat did not require additional iron. Amounts of $0.5 \mathrm{mg}$. per litre were found to be definitely detrimental. The effect of iron on toxin production has been further investigated in recent years (Pappenheimer and Johnson, 1936; Mueller, 1941 ; Mueller and Miller, 1941). These workers found that the optimal amount of iron varied greatly with the composition of the medium and with the strain. No attempt was made in the preparation of the media used in the present experiments to control either the copper or the iron content, except by the use of glass vessels and the careful control of the precipitation of the phosphates. The iron contents of the broths prepared with various peptones were determined in case the reason for the divergent results lay in this. Table I shows the total iron content of the fluid base prepared with the six peptones tested.

TABLE I

IRON CONTENT OF BROTH MEDIA PREPARED WITH DIFFERENT PEPTONES

\begin{tabular}{lcccc|c}
\hline \multicolumn{3}{c|}{ Peptone } & & Fe (mg./100 ml.) \\
\hline "Difco" proteose-peptone & $\ldots$ & 0.021 \\
Evans & $\ldots$ & $\ldots$ & $\ldots$ & $\ldots$ & 0.035 \\
"Difco" $\ldots$ & $\ldots$ & $\ldots$ & $\ldots$ & 0.086 \\
Witte's $\ldots$ & $\ldots$ & $\ldots$ & $\ldots$ & 0.050 \\
"Oxoid" $\ldots$ & $\ldots$ & $\ldots$ & $\ldots$ & 0.046 \\
"Eupeptone No. 1 ". & $\ldots$ & $\ldots$ & 0.042 \\
\hline
\end{tabular}

Except for the addition of agar these media were identical with the ones referred to above. Clearly the iron content does not explain the variation in toxin production although the best results were obtained with the two peptones showing the lowest iron values. Considerations of the iron content of the dry reagents (Table II) shows that little
TABLE II

IRON CONTENT OF DRY REAGENTS

\begin{tabular}{llll|l}
\hline \multicolumn{3}{c|}{ Reagent } & & Fe (mg./100 ml.) \\
\hline Agar powder & $\ldots$ & $\ldots$ & $\ldots$ & 5.64 \\
"Difco" proteose-peptone & $\ldots$ & 3.93 \\
Evans peptone & $\ldots$ & $\ldots$ & $\ldots$ & 4.35 \\
\hline
\end{tabular}

significance can be attached to small variations occurring in the final medium. It can be calculated that $1.5 \%$ agar corresponds to $0.084 \mathrm{mg}$. Fe. per $100 \mathrm{ml}$. For purposes of comparison this was left out of account partly because it represents a constant addition to all the media and partly as not all of it is in an ionizable form. The calculated amount of iron in $2 \%$ proteose-peptone is $0.078 \mathrm{mg}$. per $100 \mathrm{ml}$. as against the $0.021 \mathrm{mg}$. actually found. Thus, in the preparation of the medium approximately threequarters of the iron is lost with the precipitation of the phosphates. A similar loss can be calculated in the case of Evans peptone. The essential fact is that media prepared in glass vessels will yield satisfactory results without an adjustment or even determination of the iron content so long as the precipitation of the phosphates is carried out in a standard manner.

Serum Enrichment.-The remaining variable is the added sterile normal horse serum. Early in the course of these experiments it was noted that the addition of horse serum improved the reaction. This effect may well be due to enrichment of the medium leading to increased toxin production, or the adjuvant effect may be upon the flocculation, or possibly both these causes may be operative. It was assumed that even though the antitoxin in the strip might contain all the necessary non-specific constituents for flocculation it would be unlikely that they would follow the same concentration gradient as the antibody globulins. On the other hand the addition of normal serum to the medium will yield an even concentration of any nonspecific substances that may be required to intensify the flocculation lines. The views as regards the need for non-specific substances in flocculation reactions are somewhat contradictory, and the available information concerning their role and nature is insufficient for application to this test in a systematic way. It was decided, therefore, to examine the problem empirically.

Although, as has been shown, an exact reproduction of the base medium is relatively easily obtained, the addition of different batches of horse sera may materially affect results. This is clearly a considerable disadvantage for a routine test, since in the absence of knowledge of the various factors which determine the usefulness of the horse serum little can be done to obtain uniformity. Attempts to substitute bovine albumin for the horse serum were not successful. The sera used in the course of these experiments were commercially obtained No. 2 normal horse serum, heat treated, and issued for the preparation of 


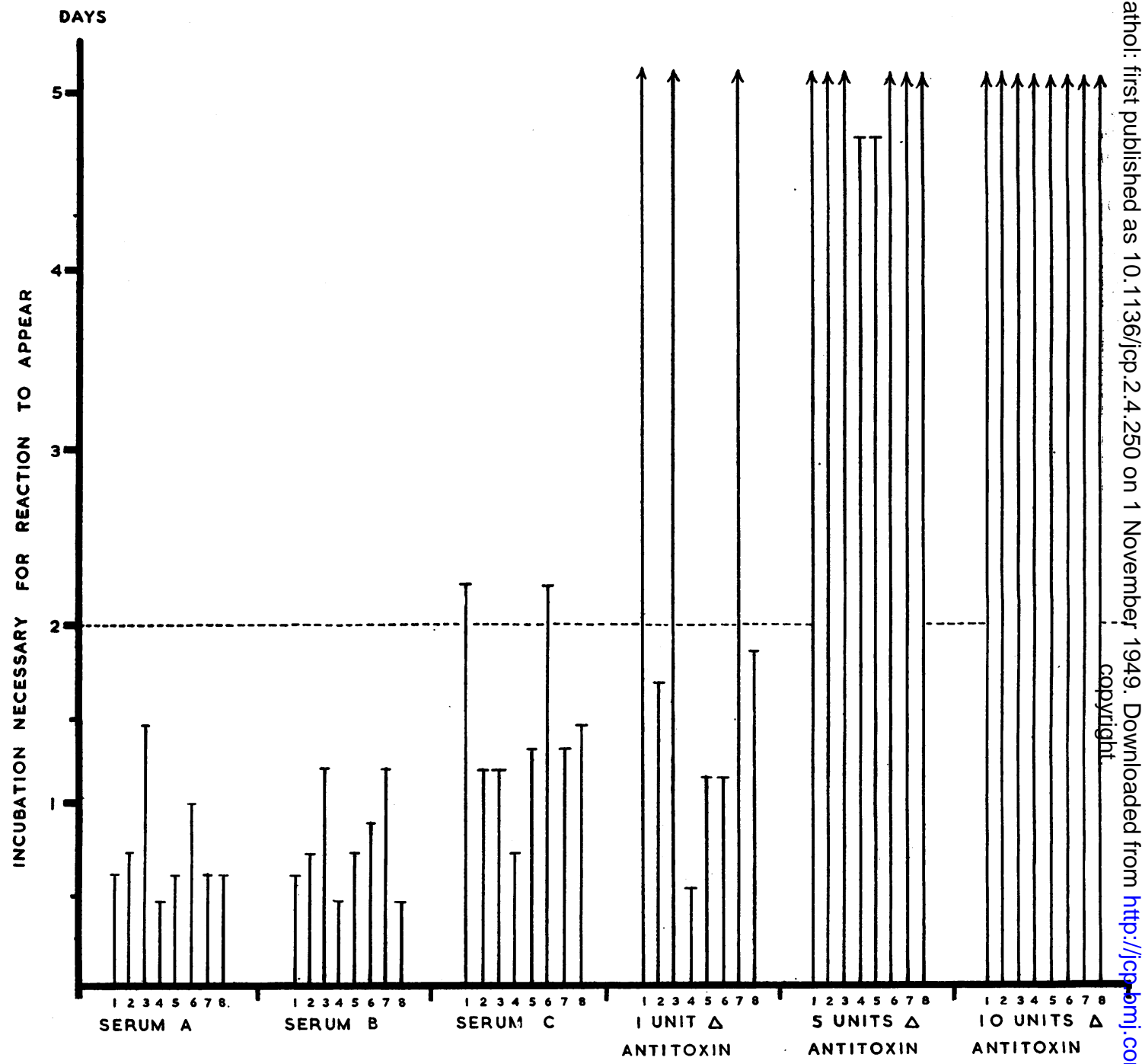

Fig. 3.-Effect of different batches of horse serum (A, B, and C) and the effect of adding diphtheria antitoxin to $₹$ serum A. Base medium prepared with "difco" proteose-peptone. Strains 1 to 8 same as in Figs. 1 and 2.

media by the Wellcome Physiological Research Laboratories. The results obtained with three different batches of horse sera are shown in Fig. 3. Two of these (A and B) were No. 2 Wellcome brand sera and the third (C) was a normal horse serum obtained from a different source. The same eight strains of diphtheria bacilli were used as in the previous experiment. The base medium was the same batch for all, made with proteose-peptone. Readings were taken as before. Sera $\mathbf{A}$ and $\mathbf{B}$ did not differ in any material way, and both yielded positives for all the strains well within 48 hours. Serum C, on the other hand, required longer incubation for the lines to develop, and two strains failed to give positive results within 48 hours, the lines becoming visible a few hours later. Clearly with this serum one would miss two $\bar{N}$ virulent strains unless readings were taken after two o days. It is hardly possible to conjecture why this $N$ serum was inferior to the others, but one possible N explanation might be that it contained natural diphtheria antitoxin which interfered with the reaction. Normal horse sera of course vary in this respect, and 0 in some unselected samples the titre may be quite $\mathbb{D}$ appreciable. It seemed interesting to test the hypo- ? thesis whether a " bad" serum could be unsatisfactory 0 on account of a high antitoxin level. To serum A $\bar{O}$ varying amounts of crude horse antitoxin were added $\stackrel{\Phi}{\circ}$

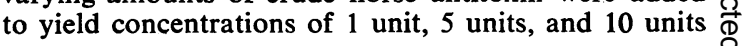


per plate. As the volume per plate was $12 \mathrm{ml}$. this corresponds to 0.08 unit, 0.42 , and 0.83 units respectively per ml. of medium. Fig. 3 shows the effect of the added antitoxin: with 1 unit per plate five strains gave positive results within $\mathbf{4 8}$ hours, but three strains, ne gravis and two intermedius, failed to produce nes even in five days. Five units per plate almost ompletely inhibited the reaction, but two strains just nanaged to produce lines within five days. It is clear rom this that a high natural antitoxin level will give in unsatisfactory serum for this test. On the other hand there is no evidence that this is the only reason or a serum proving unsatisfactory. It was found that the addition to serum $\mathrm{C}$ of purified diphtheria toxoid in amounts sufficient to over-neutralize any natural antitoxin did not improve it. This variation in the serum is at present difficult to overcome and represents the most serious disadvantage to routine use of this medium. Admittedly only small quantities of serum are required per plate, and each plate can be used for about six strains. Furthermore, a batch of serum once tested and found satisfactory can be kept in the refrigerator for long periods without apparent deterioration. The difficulty was the initial testing of the batch. At first a known strong toxin-producing strain was included on every plate, but this is obviously not satisfactory since weak toxin-producers can be overlooked in this way. Dr. A. T. Glenny suggested the use of a strain which he used for many years as a control in intracutaneous virulence tests. This strain, which he kindly let me have, was maintained in the freeze-dried state and has a fixed low virulence: in the ordinary skin tests it gives a reaction which can just be accepted as positive. On testing with the plate method it was regularly late in producing a line, and with serum $A$ it required about 40 hours' incubation for a positive reaction to appear. Two further strains were encountered which showed similar behaviour, one a gravis and the other an intermedius strain. Both of these were reported as giving a very weak intracutaneous virulence test, and this had its counterpart in a weak line appearing only towards the end of the second day's incubation. At present this biological approach constitutes the only way of standardizing the serum. Once a batch is found satifactory it can be kept at low temperature, but it is advisable to include one of the weakly toxicogenic strains with each series of tests as a control.
The Reading of the Test.-The reading of the test presents no difficulties and requires no previous experience. The lines at their earliest appearance require oblique illumination and a hand lens. Good oblique illumination can be obtained by mounting two pieces of photographic paper between half-plate glass leaving a strip of about $1 \mathrm{~cm}$. in the middle. This slit is held behind and parallel to the filter strip. By moving it slightly up or down in front of an electric bulb, a very early reading of the lines can be taken. However, a few hours later they become readily visible even by transmitted light. By the end of 24 hours the large majority of the virulent strains showed lines easily visible by transmitted light.

\section{Comparison of In Vivo and In Vitro Tests}

The final assessment of the method from the practical point of view lies in its usefulness when applied to a larger number of strains of $C$. diphtheriae and in its agreement with the accepted animal tests of virulence. When we examine the tests used as a routine for establishing whether a strain is "virulent" or not, we find that the test, subcutaneous or intradermal, is based on specific neutralization of toxin produced in vivo. The difference between the established virulence tests for diphtheria bacilli and the in vitro test described here, is only the difference between the conditions leading to toxin production. In other words, the question to be answered is whether it is possible to produce a nutrient medium catering for the individual requirements for toxin production of all the strains encountered and whether the test is sensitive enough to detect the presence of the toxin thus produced. The results of parallel tests carried out on 135 strains are shown in Table III, the same strains giving identical results by each method. These strains were mostly isolated and tested during the latter part of 1947 in London. The determinations of type and guinea-pig virulence were carried out independently of the plate tests by the bacteriologists who supplied the strains. Of the 67 strains described as gravis only one was avirulent, and this was obtained from the National Collection of Type Cultures. Of the 45 mitis strains, on the other hand, no less than 13 were avirulent, and all but one of these were isolated in the course of routine work. Of the 23 intermedius and untyped strains three were avirulent.

TABLE III

COMPARISON OF GUINEA-PIG AND PLATE VIRULENCE TESTS

\begin{tabular}{|c|c|c|c|c|c|c|c|c|c|}
\hline \multirow{2}{*}{\multicolumn{4}{|c|}{ Type of $C$. diphtheriae }} & \multicolumn{2}{|c|}{ Guinea-pig Test } & \multicolumn{3}{|c|}{ Plate Test } & \multirow[t]{2}{*}{ Total No. } \\
\hline & & & & Virulent & Avirulent & $\begin{array}{l}\text { Positive in } \\
24 \text { Hours }\end{array}$ & $\begin{array}{c}\text { Positive in } \\
48 \text { Hours }\end{array}$ & $\begin{array}{c}\text { Negative in } \\
58 \text { Hours }\end{array}$ & \\
\hline Gravis & $\cdots$ & $\cdots$ & $\cdots$ & 66 & 1 & 61 & 66 & 1 & 67 \\
\hline Mitis & $\cdots$ & $\cdots$ & $\cdots$ & 32 & 13 & 27 & 32 & 13 & 45 \\
\hline \multicolumn{4}{|c|}{ Intermedius and untyped ... } & 20 & 3 & 14 & 20 & 3 & 23 \\
\hline
\end{tabular}




\section{Discussion}

The fact that the agreement in this series was absolute suggests that the sensitivity of the two tests is of a comparable order, and this is further borne out by the findings with the weakly toxicogenic strains already referred to. This is surprising indeed as the amount of diphtheria toxin required to produce a positive skin test in a guinea-pig is extremely minute, whereas the minimum amount of toxin required to produce a visible line in the plate is considerable and amounts to about 6 units. The two tests are, however, not comparable on the basis of the quantity of toxin alone since the conditions under which the organisms grow are vastly different. In the plate technique the organisms grow unhindered and a surplus of nutrient material designed for toxin production is available so that the accumulation of toxin is continuous.

From these observations it would seem natural to connect the time of appearance of the lines with the amount of toxin produced. Clinically and epidemiologically a method giving quantitative information of an organism's toxin-producing capacity would be of value. In model experiments using one filter strip soaked in toxin and the other in antitoxin, the position and angle of the line were related to the quantities used. With a streak of inoculum, however, visible growth precedes for a variable time the formation of the toxin-antitoxin line. The position of the line formed is dependent therefore on the actual shape of the antitoxin gradient at the time when toxin begins to be produced in an appreciable concentration. It is not practicable, for routine purposes, to measure the distance of the line from the filter-strip. On the other hand it was found that a given strain will produce a line within more or less the same time on repeated examinations, and it seems that the time can be taken to reflect a biological characteristic of the strain. Thus, although it is strictly speaking not a measure of the amount of toxin formed by a given strain, it still yields information of interest concerning the toxin-producing capacity.

Twenty-nine of the 135 strains tested were obtained from the National Collection of Type Cultures. Of these 27 were found to be virulent : 20 yielded positive plate tests within 24 hours and seven $(26 \%)$ only after 48 hours' incubation. Of the remaining 106 strains, the majority of which were freshly isolated, 91 were virulent. Eightythree of these showed a positive plate reaction within 24 hours, but eight strains required 48 hours' incubation for the positive reaction to appear. This confirmed the impression that old laboratory strains require rather longer incubation. The percentage of strains requiring as long as 48 hours'은 incubation to produce a positive reaction was $26 \%$ of the old laboratory strains, but only $8.8 \%$ of the freshly isolated virulent strains. Admittedly the numbers in each group are small. It is also interesting to note that all the freshly isolated $\frac{\bar{\sigma}}{\circ}$ gravis strains were virulent, and of these 27 were positive in 24 hours, leaving only two strains that needed two days' incubation. In the group of intermedius and untyped strains six were obtained from the National Collection of Type Cultures, $\vec{\omega}$ but a further three were stock cultures. Of the remaining 14 freshly isolated strains 12 were virulent and two were not. Of the 12 virulent strains? 10 gave positive results in 24 hours and the other $r_{\tilde{N}}^{+}$ two in 48 hours. Thus of the 88 freshly isolated virulent strains 81 (over 92\%) were recognizableo as such by means of the plate technique by the end ${ }_{-}$ of the first day. It was also observed that not onlyz old stock cultures, but also freshly isolated strains? can give a sluggish reaction if the streak on the test medium is made from a culture more than 24 hours old. It is essential, therefore, to use $a_{-}^{-}$ young culture. The original culture may be on inspissated serum, blood agar, or tellurge medium.

It can be concluded from these data that the routine virulence test as performed by the intra-o dermal method, and the in vitro test here described, give complete agreement as regards the 135 strains $\varrho$ tested. This statement does not, however, invali- $\overrightarrow{0}$ date the argument that true "virulence" of diph- 3 theria bacilli is not synonymous with toxicogenicity. If there is a difference and toxin production? is only one of the criteria of virulence, then we have at present no method available to assess the sum total of the various factors entering into the 3 concept of virulence. It is clear, however, that toxin producion is a sine qua non of virulence $\frac{}{3}$ It is not possible to say whether a reversion to toxin production occurs in the case of avirulent strains. An interesting observation bearing on this question was made in the case of an old type culture strain (N.C.T.C. 322/Centry). This was received as avirulent, having been tested in $1920 \mathrm{n}$ by the bacteriologist who isolated it. It was then found that $4 \mathrm{ml}$. of a culture filtrate (further details ${ }^{\mathrm{\omega}}$ not available) were non-lethal to guinea-pigs.? This organism was found to be toxicogenic in vitro and this was confirmed by an intradermal animale test. Unfortunately the data of the original viru-lence testing were not sufficiently detailed and it is ${ }^{-}$ not possible to say whether a reversion to virulence occurred in this case. 


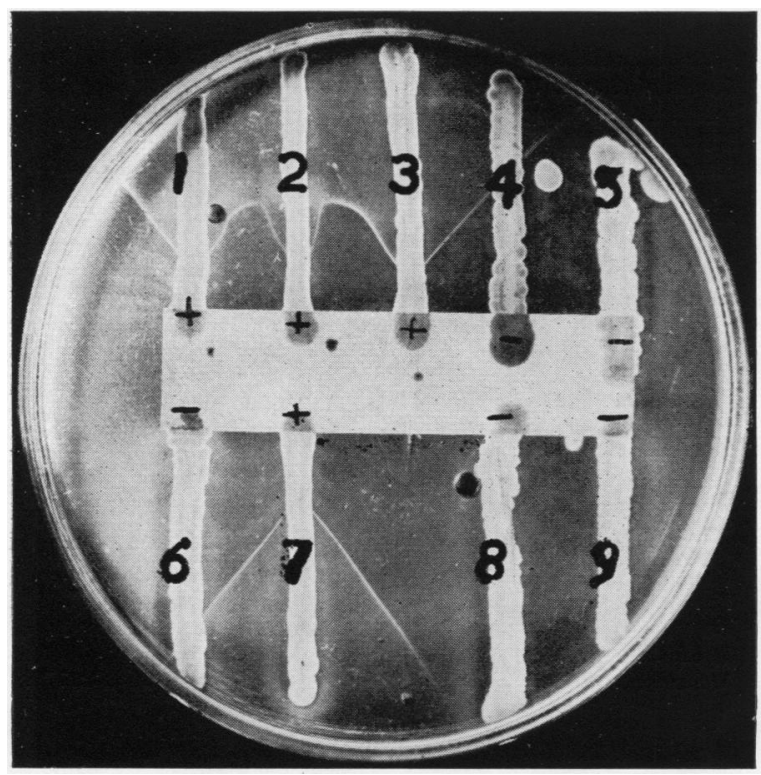

FIG. 4.-Looping of the toxin-antitoxin lines and the demonstration of non-specific turbidities. Strains 1, 2, 3 , and 7 virulent in vivo (+). Strains 4, 5, 6, 8, and 9 avirulent (-).

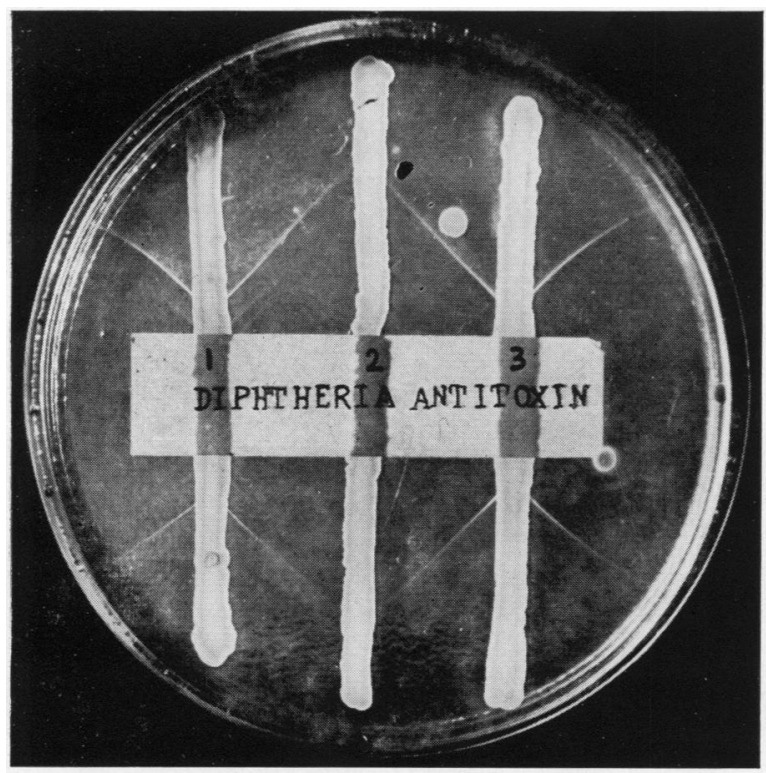

FIG. 5.-Plate photographed after prolonged incubation and several days at room temperature showing secondary lines. Strains 1 and 3 are virulent, 2 is avirulent. Strain 1 shows two fine lines developing between the toxin line and the filter strip.
The rule of looping can be made use of when determining minimal toxin production by a strain. Fig. 4 shows a plate with nine strains of which Nos. 1, 2, 3, and 7 were virulent ( + ) and Nos., 4, $5,6,8$, and 9 avirulent $(-)$ in vivo. Strain No. 2 is weakly toxicogenic and the lines produced by strains No. 1 and No. 3 show marked bending on approaching it. When the lines approach a strain that is devoid of toxin production they remain straight and cut the non-toxic streak ; that is, the line of strain No. 3 cuts across strain No. 4 which is non-virulent. It should be stressed, however, that the looping effect only applies when both lines are simultaneously produced. If one lags behind an angular joining of the lines occurs, as seen between strains No. 2 and No. 3, and this is due to the rearrangement of the antitoxin gradient around the line first formed. Any turbidity around the streak should be disregarded, since many organisms produce non-specific turbidities in serum agar media. Thus strain No. 7 , which is virulent, produces a halo, but so does strain No. 8 as well although it is avirulent.

When the incubation is prolonged beyond 48 hours, or the plates left at room temperature for several days, very fine lines showing the typical arrowhead form may appear (Fig. 5, strain No. 1). These lines are definitely not due to toxin production as they appear with both virulent and avirulent strains. To avoid confusion the plate test must not be read later than 48 hours. The characteristics of these secondary lines are as follows: they appear late, usually after several days, they are fine, and may number two or three. Apparently they are due to diffusible antigens produced by diphtheria bacilli, the exact nature of which requires further elucidation.

In view of the secondary lines it is most important that the medium used in the plate test should be capable of demonstrating minimal amounts of toxin production in 48 hours, that is, before the appearance of secondary lines might lead to confusion. The medium, the preparation of which is here described, satisfies this requirement.

\section{Summary and Conclusions}

A method is described for assessing the value of various constituents in the preparation of media for the diphtheria plate virulence test.

Six different brands of peptone were found to vary in their suitability for the test.

The iron content of the media required no adjustment provided the preparation followed the routine described. 
Batches of horse sera vary in their suitability for the test and the value of the serum used has to be established empirically.

A series of 135 strains gave identical results by the plate test and the intradermal guinea-pig test. The sensitivities of the two tests were found to be of the same order.

Over $92 \%$ of freshly isolated virulent strains gave a positive reaction within 24 hours with the plate test, and $100 \%$ were positive after 48 hours' incubation. Readings must not be taken later than 48 hours.

I wish to express my sincere thanks to Dr. A. G. Signy, Dr. E. Straker, Dr. A. Beck, and Mr. H. Proom for supplying various strains; to Dr. J. E. McCartney and Mr. J. C. Monckton for virulence-testing as well as supplying strains; to Professor T. Crawford for valuable help in the preparation of the paper, and to Mr. T. Shaw and Mr. T. Pringle for technical assistance.

\section{REFERENCES}

Ando, K., and Komiyama, T. (1935). J. Immunol., 28, 345. Carter, H. S., and Wilson, W. (1949). Glasg. med. J., 30, 43. Davis, L., and Ferry, N. S. (1919). J. Bact., 4, 217.

Elek, S. D. (1948). Brit. med. J., 1, 493.

Hazen, E. L., and Heller, G. (1931). Proc. Soc. exp. Biol. N.Y., 28

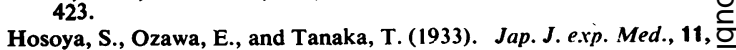
463.

Locke, A., and Main, E. R. (1928). J. infect. Dis., 43, 41.

Locke, A., and Main, E. R. (1930). J. infect. Dis., 46, 393.

Mueller, J. H. (1941). J. Immunol., 42, 343.

Mueller, J. H., and Miller, P. A. (1941). J. Immunol., 40, 21.

Ouchterlony, O. (1948). Acta path. microbiol. scand., 25, 186.

Ouchterlony, O. (1939). Lancet, 1, 346.

Park, W. H., and Williams, A. W. (1896). J. exp. Med., 1, 164.

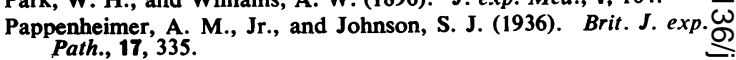

Petrie, G. F., and Steabben, D. (1943). Brit. med. J., 1, 377.

Pope, C. G. (1932a). Brit. J. exp. Path., 13, 207.

Pope, C. G. (1932b). Brit. J. exp. Path., 13, 218.

Pope, C. G., and Healey, M. (1933a). Brit. J. exp. Path., 14, 77.

Pope, C. G., and Healey, M. (1933b). Brit. J. exp. Path., $14,87$.

Ramon, G. (1929). C.R. Acad. Sci., Paris, 189, 718.

Smith, T. (1899). J. exp. Med., 4, 373.

Wadsworth, A.,'and Wheeler, M. W. (1928.) J. infect. Dis., 42, 179. 